\title{
Fabrication of p-type porous GaN on silicon and epitaxial GaN
}

O. V. Bilousov, H. Geaney, J. J. Carvajal, V. Z. Zubialevich, P. J. Parbrook, A. Giguère, D. Drouin, F. Díaz, M. Aguiló, and C. O'Dwyer

Citation: Appl. Phys. Lett. 103, 112103 (2013); doi: 10.1063/1.4821191

View online: http://dx.doi.org/10.1063/1.4821191

View Table of Contents: http://aip.scitation.org/toc/apl/103/11

Published by the American Institute of Physics

\section{Articles you may be interested in}

MBE-grown 232-270 nm deep-UV LEDs using monolayer thin binary GaN/AIN quantum heterostructures Applied Physics Letters 110, 041108 (2017); 10.1063/1.4975068

Physics and polarization characteristics of $298 \mathrm{~nm}$ AIN-delta-GaN quantum well ultraviolet light-emitting diodes Applied Physics Letters 110, 071103 (2017); 10.1063/1.4976203

Utilisation of $\mathrm{GaN}$ and InGaN/GaN with nanoporous structures for water splitting

Applied Physics Letters 105, 223902 (2014); 10.1063/1.4903246

The effect of a varied $\mathrm{NH}_{3}$ flux on growth of AIN interlayers for InAIN/GaN heterostructures

Applied Physics Letters 103, 081602 (2013); 10.1063/1.4818645

Gallium vacancies and the yellow luminescence in GaN

Applied Physics Letters 69, 503 (1998); 10.1063/1.117767

GaN, AIN, and InN: A review

Journal of Vacuum Science \& Technology B: Microelectronics and Nanometer Structures Processing, Measurement, and Phenomena 10, 1237 (1998); 10.1116/1.585897

\section{AIP| Applied Physics} Letters

Save your money for your research. potential to shape the future of It's now FREE to publish with us no page, color or publication charges apply. 


\title{
Fabrication of p-type porous GaN on silicon and epitaxial GaN
}

\author{
O. V. Bilousov, ${ }^{1}$ H. Geaney, ${ }^{2,3}$ J. J. Carvajal, ${ }^{1, a)}$ V. Z. Zubialevich ${ }^{3}$ P. J. Parbrook, ${ }^{3,4}$ \\ A. Giguère, ${ }^{5}$ D. Drouin, ${ }^{5}$ F. Díaz, ${ }^{1}$ M. Aguiló, ${ }^{1}$ and C. O'Dwyer ${ }^{2,3, b)}$ \\ ${ }^{1}$ Física i Cristallografia de Materials i Nanomaterials (FiCMA-FiCNA) and EMaS, \\ Universitat Rovira i Virgili (URV), Marcelli Domingo s/n, E-43007 Spain \\ ${ }^{2}$ Department of Chemistry, University College Cork, Cork, Ireland \\ ${ }^{3}$ Tyndall National Institute, Lee Maltings, Cork, Ireland \\ ${ }^{4}$ Department of Electrical \& Electronic Engineering, University College Cork, Cork, Ireland \\ ${ }^{5}$ Interdisciplinary Institute for Technical Innovation, Université de Sherbrooke, Sherbrooke, \\ Quebec JIK 2RI, Canada
}

(Received 31 July 2013; accepted 29 August 2013; published online 11 September 2013)

\begin{abstract}
Porous GaN layers are grown on silicon from gold or platinum catalyst seed layers, and self-catalyzed on epitaxial GaN films on sapphire. Using a Mg-based precursor, we demonstrate p-type doping of the porous GaN. Electrical measurements for p-type GaN on Si show Ohmic and Schottky behavior from gold and platinum seeded GaN, respectively. Ohmicity is attributed to the formation of a $\mathrm{Ga}_{2} \mathrm{Au}$ intermetallic. Porous p-type $\mathrm{GaN}$ was also achieved on epitaxial n-GaN on sapphire, and transport measurements confirm a p-n junction commensurate with a doping density of $\sim 10^{18} \mathrm{~cm}^{-3}$. Photoluminescence and cathodoluminescence confirm emission from $\mathrm{Mg}$-acceptors in porous p-type GaN. (C) 2013 AIP Publishing LLC. [http://dx.doi.org/10.1063/1.4821191]
\end{abstract}

Gallium nitride $(\mathrm{GaN})$ is considered one of the most important wide band-gap semiconductors for a number of applications in electronics and optoelectronics. ${ }^{1}$ In its porous form, $\mathrm{GaN}$ has received particular interest due to beneficial optical and electronic properties for gas sensors with high sensitivity $^{2}$ and light-emitting diodes (LEDs) with high light extraction efficiency. ${ }^{3}$ The formation of p-type porous GaN is particularly relevant ${ }^{4}$ for the fabrication of junctions and active regions for GaN-based LEDs with improved light extraction efficiency and external quantum efficiencies by gradation of the effective refractive index. ${ }^{5}$ Porous GaN has been typically fabricated by (photo)electrochemical and chemical etching methods, ${ }^{6-9}$ predominantly a top-down etching of epitaxial GaN, giving textured surfaces as a result of pore coalescence and variations in etch rates for extended etching times. A range of nanoscale III-N materials ${ }^{10,11}$ in arrays and assemblies spanning emission from the blue to red regions of the visible spectrum, ${ }^{12}$ and white light emitting light emitting devices and multi quantum wells have also been realized. ${ }^{13}$ The ability to form relatively uniform, crystalline porous $\mathrm{GaN}$ layers over large areas without unwanted surface damage through complex etching without photolithographic masking or processing is challenging. Previously, we reported the bottom-up growth of lowresistivity, Ohmically contacted porous $\mathrm{n}-\mathrm{GaN}$ in a single growth step ${ }^{14}$ without the need for any secondary etching or chemical treatment after growth to induce porosity. ${ }^{15,16}$

$\mathrm{Mg}$ is the dopant most commonly used to generate p-type conductivity in GaN. Mg substitution of Ga in the GaN lattice is the shallowest acceptor which can be introduced in sufficient concentrations to enable p-type conduction.

In this letter, we detail the growth of porous $\mathrm{GaN}$ on $\mathrm{Si}$ and epitaxial GaN substrates on sapphire and evidence the introduction of p-type character by $\mathrm{Mg}$-doping. Porous $\mathrm{GaN}$

\footnotetext{
a) joanjosep.carvajal@urv.cat

b)c.odwyer@ucc.ie
}

formation was conducted within a chemical vapor deposition (CVD) system with growth on Si proceeding via the use of either a Pt or Au catalyst layer. Electrical transport measurements for the porous p-type $\mathrm{GaN}$ grown on $\mathrm{Si}$ from $\mathrm{Au}$ and Pt show Ohmic and Schottky behavior, respectively, with the former attributed to the formation of an $\mathrm{Au} / \mathrm{Ga}$ intermetallic. Porous p-type GaN growth was also achieved on non-porous n-type epitaxial $\mathrm{GaN}$ and electrical transport measurements were used to confirm the formation of a $p-n$ junction commensurate with the formation of p-type GaN. Additional evidence for the formation of p-type $\mathrm{GaN}$ was also obtained from low temperature photoluminescence (PL) data which showed clear differences for the undoped and Mg-doped samples.

$\mathrm{GaN}$ doped with $\mathrm{Mg}$ was produced through the direct reaction of metallic Ga with $\mathrm{NH}_{3}$ in a simple CVD system. Nanoporous GaN microparticles were deposited on silicon (100) substrates with an area of $1 \mathrm{~cm}^{2}$ coated with $20 \mathrm{~nm}$ thick films of $\mathrm{Au}$ or Pt used as the growth catalyst in a horizontal tubular furnace Thermolyne 79300 (see schematics in Figure 1). Further details can be found elsewhere. ${ }^{14,15} \mathrm{Mg}$ doped samples were annealed in atmosphere of $\mathrm{N}_{2}$ for $20 \mathrm{~min}$ in order to break $\mathrm{Mg}-\mathrm{H}$ complexes and activate the p-type conductivity of porous GaN. The epitaxial GaN substrates had n-type (doped with $\mathrm{Si}$ ) conductivity. Mg-doped porous GaN was also grown on epitaxial n-type GaN substrates on sapphire. Gallium metal (99.99\%), ammonia ( $>99.98 \%)$, and magnesium nitride $(99.95 \%)$ were used as the $\mathrm{Ga}, \mathrm{N}$, and $\mathrm{Mg}$ sources, respectively, with the magnesium source placed $4 \mathrm{~cm}$ up-stream of the Ga source. The porous GaN layers were imaged using a JEOL JSM 6400 scanning electron microscope (SEM). PL was excited by $244 \mathrm{~nm}$ emission (second harmonic of $488 \mathrm{~nm}$ line) from a cw Ar-ion laser with power density of $2 \mathrm{~W} / \mathrm{cm}^{2}$. PL spectra were acquired using a Horiba iHR320 spectrometer equipped with a Synapse CCD matrix. Samples were placed in an evacuated chamber of a Janis closed-cycle helium cryostat 
(a)
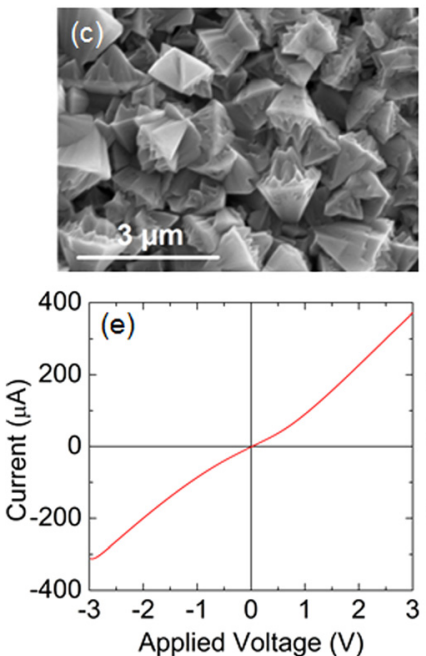

Applied Voltage (V)

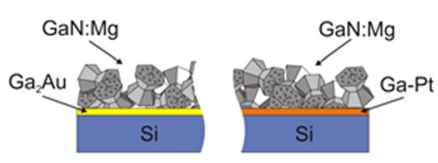

(b)
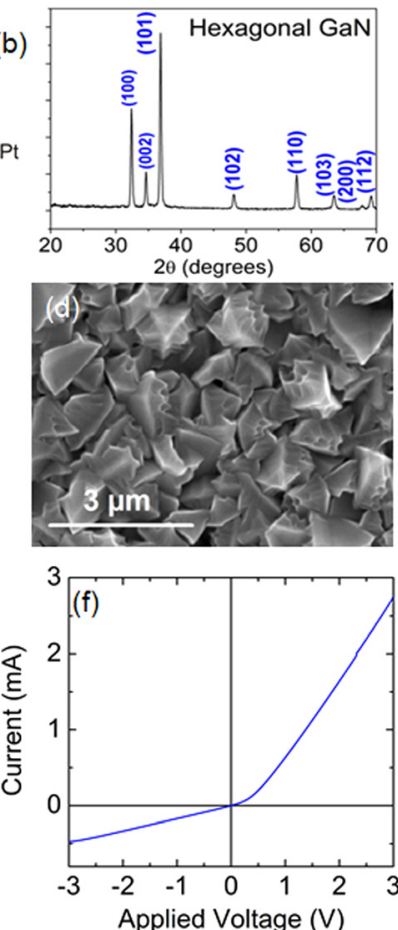

FIG. 1. (a) Schematic presentation of porous $\mathrm{Mg}$-doped $\mathrm{GaN}$ on $\mathrm{Si}$ with $\mathrm{Ga}_{2} \mathrm{Au}$ and Pt-Ga intermetallic layer, (b) X-ray diffraction pattern of porous $\mathrm{Mg}$ doped $\mathrm{GaN}$ on $\mathrm{Si}$; $\mathrm{SEM}$ images of porous $\mathrm{Mg}$-doped $\mathrm{GaN}$ grown on $\mathrm{Si}$ substrates coated with (c) Au and (d) Pt and corresponding I-V curves for (e) Au- and for (f) Pt-seeded GaN.

for PL measurements at different temperatures. Room temperature cathodoluminescence (CL) imaging and spectroscopy were performed in a field emission scanning electron microscope (Zeiss Supra 55) operating at 5-10 keV and 200 pA using a Gatan MonoCL 2 system and a Hamamatsu photomultiplier tube (R2228) over a scanned area of $4000 \mu \mathrm{m}^{2}$. All spectra were corrected for the monochromator and detector response and normalized to maximum intensity. $\mathrm{X}$-ray diffraction (XRD) measurements were made using $\mathrm{Cu}$ $\mathrm{K} \alpha$ radiation in a Bruker-AXS D8-Discover diffractometer operated at $40 \mathrm{kV}$ and $40 \mathrm{~mA}$, equipped with parallel incident beam (Göbel mirror), a General Area Diffraction Detector System (GADDS), and a $500 \mu \mathrm{m}^{2} \mathrm{X}$-ray collimator system.

Figure 1(a) shows schematic presentation of porous $\mathrm{GaN}$ on $\mathrm{Si}$ with $\mathrm{Ga}_{2} \mathrm{Au}$ and Pt-Ga intermetallic layers. The XRD pattern shown in Figure 1(b) for $\mathrm{Mg}$ doped GaN confirms crystalline wurtzite GaN. Figures 1(c) and 1(d) confirm that the porous GaN particles have a characteristic morphology consisting of faceted crystals with a mean size of $\sim 1.5 \mu \mathrm{m}$, similar to that observed in undoped samples. ${ }^{14}$ In both cases, crystal growth occurs progressively on the surface with individual crystals until a layer of porous $\mathrm{GaN}$ microcrystals covers the surface as a porous layer.

Two-probe electrical measurements of the various $\mathrm{Mg}$ doped GaN samples were conducted using In/Ga liquid eutectic contacts and a Biologic SP-50 potentiostat. An In/Ga eutectic droplet was used as the Ohmic contact. Linear voltage sweeps were obtained between the range of $-3 \mathrm{~V}$ and $3 \mathrm{~V}$ with a $50 \mathrm{mV} / \mathrm{s}$ sweep rate. Figures $1(\mathrm{e})$ and $1(\mathrm{f})$ show I-V curves taken from $\mathrm{Mg}$-doped porous $\mathrm{GaN}$ grown from $\mathrm{Au}-$ and Pt-coated Si (100) substrates, respectively. For samples grown using an Au catalyst, a near Ohmic contact behavior was repeatedly observed which is consistent with the formation of $\mathrm{a} \mathrm{Ga}_{2} \mathrm{Au}$ intermetallic between the underlying substrate and $\mathrm{GaN}$ material ${ }^{9}$ in a similar way observed for undoped porous GaN particles. ${ }^{14}$ However, for the GaN samples grown using Pt as catalyst, the response was markedly different with the profile indicating the formation of a Schottky contact. For porous $\mathrm{GaN}$ specifically, the thickness is determined by the size of the crystals and their assembly on the surface, which results in a "rough" topology, with a high density of grain boundary scattering centers. For electrical measurements, particle distributions ${ }^{14}$ confirm that both nearest neighbour distances and overall distribution of various nonspherical catalyst material prevent the formation of a percolating conduction layer beneath the GaN; the electrical characterization is of the porous GaN polycrystalline layer. At room temperature, the effective linearity of the $\mathrm{I}-\mathrm{V}$ response measured for porous GaN to high work function metals, with an intermetallic seed layer between the metal and GaN, is maintained at high bias. As stated earlier, the Pt-Ga alloy results in a Schottky contact to the porous p-type GaN. The Schottky barrier height $\varphi_{B, p}^{o}$ $(\mathrm{SBH})$ can be estimated from $I=I_{0} \exp \left[\left(q V-I R_{s}\right) / k T-1\right]$ with $I_{0}=A A^{* *} T^{2} \exp \left(-q \varphi_{B, p}^{o} / k T\right)$, where $A^{* *}$ is the effective Richardson constant $\left(26.4 \mathrm{~A} \mathrm{~cm}^{-2} \mathrm{~K}^{-2}\right) .{ }^{17}$ The estimated $\mathrm{SBH}$ for $\mathrm{Pt}$ contacted porous $\mathrm{p}-\mathrm{GaN}$ is $0.57 \mathrm{eV}$. While a small in-built potential also exists for the Au-contacted sample, the symmetry in the response at negative bias confirms that the presence of the intermetallic $\mathrm{Ga}_{2} \mathrm{Au}$ at the $\mathrm{GaN}-\mathrm{Si}$ interface prevents a rectifying barrier. Also, as with unintentionally n-type GaN growth we investigated previously, ${ }^{14}$ the $\mathrm{Pt}-\mathrm{Ga}$ contact to the $\mathrm{GaN}$ at the silicon interface results in an order of magnitude higher current, but in the case of $\mathrm{p}-\mathrm{GaN}$, the transport mechanism gives a non-linear, Schottky-type response.

To further investigate the nature of the Mg-doped porous $\mathrm{GaN}$, it was grown on epitaxial n-type $\mathrm{GaN}$ substrates (without the presence of catalyst layer) under the same conditions. In this case, the porous crystals of GaN have a different morphology as the porous GaN was found to be grown oriented along the c-axis direction with respect to the epitaxial GaN on the substrate (Figures 2(a) and 2(c)). Figure 2(b) shows I-V measurements taken between the underlying epitaxial n-type GaN substrate and the porous p-type GaN of porous contacts; the clear $\mathrm{p}-\mathrm{n}$ junction response confirms the formation of p-type porous GaN confirmed by the asymmetric diode response in Fig. 2(d) where a clear exponential I-V relationship is found. The high bias resistance turn-over of the porous p-type GaN occurs at $\sim 1 \mathrm{~V}$. At this voltage, the exponential current increase results in high level carrier injection across the junction as the voltage is increased, and then follows a linear dependency on applied voltage above $1 \mathrm{~V}$ (and is found to remain so up to $\sim 5 \mathrm{~V}$ in all cases), consistent with an effective series resistance which is believed to arise from the high density of inter-crystal contacts within the porous $\mathrm{GaN}$ layer. These values are consistent with corresponding characteristic voltages found in uniform epitaxially grown GaN. ${ }^{18}$

The barrier to the exponential current increase is found to be much lower than the expected GaN diode response, i.e., $E_{\mathrm{g}} / q \sim 2.16 \mathrm{~V}$. The knee voltage where significant 
(a)

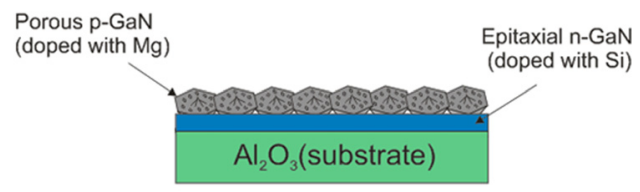

(b)

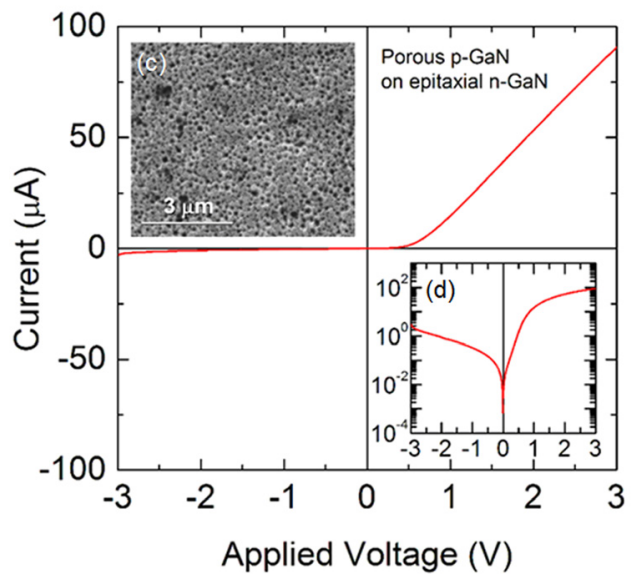

FIG. 2. (a) Schematic presentation of porous GaN on epitaxial GaN substrate. (b) I-V curve of the p-n junction formed between porous $\mathrm{GaN}$ doped with $\mathrm{Mg}$ and n-type epitaxial GaN. (c) Top-view SEM image of the porous GaN layer. (d) $\ln I(V)$ curve of the porous p-GaN corresponding to the I-V curve in (b).

current is seen is found to be in the range of $E_{\mathrm{g}} / 6 q-E_{\mathrm{g}} / 4 q$, i.e., $0.36-0.54 \mathrm{~V}$. This response has several features that have been seen in InN and GaN nanowires arrays but not in bulk or thin film GaN. ${ }^{19}$ However, the p-type nature of the porous $\mathrm{GaN}$ is confirmed and in both cases, low reverse bias leakage current are found for the porous assembly of GaN crystals in the layer.

The effective hole density is estimated at $300 \mathrm{~K}$ for the porous $\mathrm{GaN}$ layer from $n p=N_{C} N_{V} \exp \left(-E_{g} / k T\right)$, where $N_{\mathrm{C}}=4.3 \times 10^{14} \cdot T^{3 / 2} \mathrm{~cm}^{-3}$ and $N_{\mathrm{V}}=8.9 \times 10^{15} \cdot T^{3 / 2} \mathrm{~cm}^{-3}$ are the effective densities of states of the conduction and valence bands, using the effective hole and electron masses for wurtzite $\mathrm{GaN}$ at $300 \mathrm{~K} .{ }^{20}$ Using $n=10^{19} \mathrm{~cm}^{-3}$ for the epitaxial n-GaN, we estimate the hole density for the porous $\mathrm{p}-\mathrm{GaN}$ to be $\sim 9 \times 10^{18} \mathrm{~cm}^{-3}$. From the Poisson distribution of space charge distribution leading to the dependence of majority and minority carrier densities across an abrupt, one-side junction, the barrier potential $V_{\mathrm{B}}$ across the depletion region for the porous $\mathrm{GaN}$ junction is estimated as $V_{B}=k T / q \ln \left(N_{D} N_{A} / n_{i}^{2}\right)=0.9 \mathrm{~V}$, where $N_{D}$ and $N_{A}$ are the donor and acceptor densities, respectively, determined for the charge neutrality condition $p+N_{D}^{+}=n+N_{A}^{-}$, and $n_{i}^{2}=2.8 \times 10^{11} \mathrm{~cm}^{-3}$ is the intrinsic doping density of GaN estimated by the $e-h$ product above. The value (at zero applied bias) is greater $\left(E_{\mathrm{g}} / 2.5 q\right)$ than the knee voltage, although typically found for epitaxial GaN with knee voltages in p-n junctions $>2 \mathrm{~V}$. This discrepancy is likely due to a graded junction and similar to other nanoscale III-Ns that show similar behaviour but for porous $\mathrm{GaN}$ does not seem to be a dominant size effect (micron sizes crystals).

Cathodoluminescence investigations of the Mg-doping of porous $\mathrm{GaN}$ was performed using two different quantities of dopant precursor. Figure 3(a) shows SEM and CL images of porous $\mathrm{n}-\mathrm{GaN}$ crystals within the porous layer. Porous GaN allows a considerable signal to be observed for the band-edge emission compared with that at $V_{a c c}=20 \mathrm{kV}^{21}$

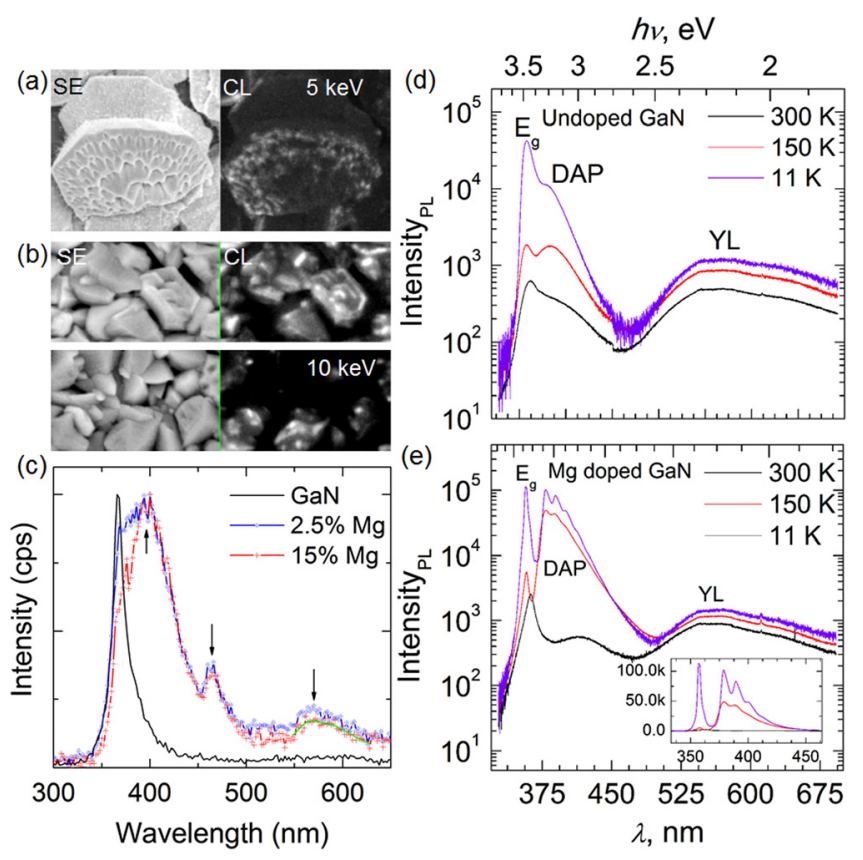

FIG. 3. (a) Secondary electron (SE) and CL images of n-GaN crystals at $5 \mathrm{kV}$. (b) $\mathrm{SE}$ and $\mathrm{CL}$ images of porous $\mathrm{GaN}$ with $2.5 \%$ and $15 \% \mathrm{Mg}$ precursor at $10 \mathrm{kV}$. (c) Cathodoluminescence spectra from Mg-doped porous GaN crystals before and after Mg-doping. (d) Temperature-dependent PL spectra of undoped and (e) $\mathrm{Mg}$-doped $\mathrm{GaN}$ acquired after excitation at $325 \mathrm{~nm}(3.8 \mathrm{eV})$. The inset shows the PL emission on a linear scale.

Typically, epitaxial or MBE grown GaN films suffer from low intensities due to $e$ - $h$ pair formation at low voltages which increases surface recombination that is non-radiative. In this case of porous $\mathrm{GaN}$, the emission is enhanced at the high density of sharp edge surfaces found on the crystal and thus throughout the porous film. Figure 3(b) shows a pair of SEM and CL images of porous GaN doped with $2.5 \%$ and $15 \% \mathrm{Mg}$ and their corresponding CL spectra (Fig. 3(c)) recorded at room temperature. In the spectra acquired at an accelerating voltage, $V_{a c c}=10 \mathrm{kV}$, a shift of the CL peak is found in comparison to undoped GaN. The yellowluminescence (YL) seen from CL shows two contributions, typically ascribed to pair recombination and deep level emission characteristic of doped p-type GaN. The near-band edge emission is similar in both doped samples and not found in undoped $\mathrm{GaN}$, but lower in energy $(397 \mathrm{~nm})$ than the bandgap emission from $\mathrm{GaN}$ (the monochromator edge was $368 \mathrm{~nm}$ for $\mathrm{Mg}$-doped $\mathrm{CL}$ ).

PL measurements were performed on both Mg-doped samples and undoped porous GaN to conclusively define the p-type character of the crystals. Figures 3(d) and 3(e) show PL spectra taken at 11,150 , and $300 \mathrm{~K}$ at a constant laser power density. We observe that the undoped GaN crystal forming the porous layer exhibit expected bandedge luminescence, blue-shifting from $3.43 \mathrm{eV}$ at $300 \mathrm{~K}$ to $3.46 \mathrm{eV}$ at $11 \mathrm{~K}$ with a narrow FWHM of $\sim 12 \mathrm{meV}$. Emission from high energy bound excitons sometimes observed in epitaxially grown GaN (of any conduction type) is not found in these samples. The capture of excitons by deep energy centers often from localized states accompanying defects in epitaxial GaN films drastically limit the radiative recombination near the band gap, which is detrimental for device function. The near band-gap 
luminescence in Figure 3(d) is quite broad for undoped GaN crystal forming the porous layer. ${ }^{22}$ None of the constituent phases at the silicon interface ( $\mathrm{Pt}, \mathrm{Au}$, their alloys, etc.) contribute to the luminescence. The blue luminescence is often attributed to recombination between a deep donor and a $\mathrm{Mg}$ acceptor. ${ }^{23,24}$ Broad emission near the band gap typically results from tailing of the density of states due to randomly distributed impurities, but the emission bandwidth reduces at high doping densities. We find that the near-band gap emission and band-edge emission equilibrate in intensity at $150 \mathrm{~K}$, but the band-edge emission dominates at higher and lower temperatures.

The PL spectrum of the porous GaN crystals after activation of $\mathrm{Mg}$ conclusively demonstrates $\mathrm{Mg}$ incorporation as an acceptor state in the $\mathrm{GaN}$ lattice, resulting in the p-type character confirmed through I-V measurements in p-n junctions. The room temperature band-edge emission is marginally lower energy at $3.41 \mathrm{eV}$ compared with the n-type material, but this emission is located at a consistent value of $3.46 \mathrm{eV}$ at temperatures down to $11 \mathrm{~K}$ with a narrow FWHM of $\sim 5 \mathrm{meV}$. The band gap emission in both materials is over an order of magnitude more intense at $11 \mathrm{~K}$ compared with $150 \mathrm{~K}$. We do observe some emission at very high superbandgap energies of $\sim 3.5 \mathrm{eV}$, consistent with a free or shallow-bound exciton. As the $\mathrm{GaN}$ is $\mathrm{Mg}$ doped and neither Si nor shallow $\mathrm{O}$ donor-bound excitons are likely, we attribute this weak emission to acceptor bound excitons linked to $\mathrm{Mg}$ incorporation. Deeply bound-exciton emission is not observed from the substrate-GaN interface and no reduction in band-edge energy is found due to bound excitons linked to atomic vacancies.

The band-edge luminescence is followed by the donoracceptor pair (DAP) luminescence at 3.27 and $3.28 \mathrm{eV}$ at 150 and $11 \mathrm{~K}$, respectively. This DAP emission is not found at $300 \mathrm{~K}$ for the p-type porous $\mathrm{GaN}$. This type of emission has been previously observed and attributed to the formation of a $\mathrm{Mg}$ complex or some native defect level. As expected from $\mathrm{Mg}$ acceptors in doped $\mathrm{GaN}$, we find the longitudinal optical (LO) phonon replicas at 3.18 and $3.19 \mathrm{eV}$ at 150 and $11 \mathrm{~K}$, respectively, with several harmonics observable at $11 \mathrm{~K}$, indicating that the electronic quality of the doped GaN crystals directly on $\mathrm{Si}$ matches that of epitaxially grown $\mathrm{p}$ doped $\mathrm{GaN}^{25}$

Yellow luminescence (YL) in $\mathrm{n}-\mathrm{GaN}$ is normally attributed to point defect $\mathrm{Ga}$ vacancies, impurities, such as oxygen and carbon. ${ }^{26} \mathrm{YL}$ is also present in Mg-doped GaN, as observed in Figure 3(e). The marked intensity variation in band-edge and YL as a function of temperature is seen on a linear scale in the inset. Most probably, the YL arises from a transition between the conduction band or shallow donors ${ }^{27}$ and deep acceptor levels caused by crystallographic point defects in the GaN crystals $^{28}$ since we find that the YL emission profile and temperature-dependence is similar and characteristic of both undoped and p-type $\mathrm{GaN}$ crystals comprising the porous GaN layer. The YL is weakly temperature-dependent and is not found to vary considerably due to $\mathrm{Mg}$ incorporation into the lattice.

In summary, we have shown that high structural and electronic quality p-type GaN can be grown as single crystals directly on $\mathrm{Si}$ and also on epitaxial $\mathrm{GaN}$ substrates on sapphire as a porous layer. On $\mathrm{Si}$, vapor-solid-solid growth catalyzed by either Au or Pt allows GaN growth with the direct formation (in the case of $\mathrm{Au}$ ) of an Ohmic intermetallic contact, without unwanted effects of interfacial resistive oxide formation nor the need for post-deposition annealing steps to introduce Ohmicity. Self-catalyzed growth of porous p-type $\mathrm{GaN}$ is also demonstrated to be possible on sapphire and grows with a similar porous morphology. P-n junction measurements of porous $\mathrm{GaN}$ and epitaxial $\mathrm{GaN}$ show that $\mathrm{Mg}$ incorporation from the precursor results in $\mathrm{p}$ type conduction character. The overall method of using intermetallic as Ohmic contacts could also be applied to simultaneously grow and contact nanoscale III-N compounds and nanoscale arrays of $\mathrm{p}-\mathrm{n}$ junctions for optoelectronic devices, and on epitaxial GaN, this extends to $\mathrm{p}-\mathrm{n}$ junctions of porous crystalline assemblies that are strongly $\mathrm{c}$-axis aligned with the underlying opposite conduction type GaN substrate. Porous III-N materials with rational doping also have great potential for wide band-gap biosensors.

This work was supported by the EU Framework 7 under Project No. FP7-SPA-2010-263044, the Spanish Government under Projects No. MAT2011-29255-C02-02, TEC201021574-C02-02, and by Catalan Authority under Project No. 2009SGR235. This work was also supported by the UCC Strategic Research Fund, by Science Foundation Ireland under Award No. 07.SK.B1232a-STTF11, and through the Irish Research Council New Foundations Award 2012.

${ }^{1}$ S. Nakamura, S. Pearton, and G. Fasol, The Blue Laser Diode: The Complete Story (Springer-Verlag, Berlin Heidelberg, 2000).

${ }^{2}$ A. Ramizy, Z. Hassan, and K. Omar, Sens. Actuators B 155(2), 699-708 (2011).

${ }^{3}$ C.-F. Lin, C. Kuei-Ting, L. Chun-Min, and Y. Chung-Chieh, IEEE Electron Device Lett. 30(10), 1057-1059 (2009).

${ }^{4}$ S. J. Pearton and F. Ren, Adv. Mater. 12, 1571 (2000).

${ }^{5}$ C.-C. Yang, C. F. Lin, C.-M. Lin, C. C. Chang, K.-T. Chen, J.-F. Chien, and C.-Y. Chang, Appl. Phys. Lett. 93(20), 203103-203103-203103 (2008).

${ }^{6}$ Y. D. Wang, S. J. Chua, M. S. Sander, P. Chen, S. Tripathy, and C. G. Fonstad, Appl. Phys. Lett. 85(5), 816-818 (2004).

${ }^{7}$ S. Y. Bae, H. W. Seo, J. Park, H. Yang, and B. Kim, Chem. Phys. Lett. 376(3), 445-451 (2003).

${ }^{8}$ D. J. Diaz, T. L. Williamson, I. Adesida, P. W. Bohn, and R. J. Molnar, J. Appl. Phys. 94(12), 7526-7534 (2003).

${ }^{9}$ J. A. Bardwell, I. G. Foulds, J. B. Webb, H. Tang, J. Fraser, S. Moisa, and S. Rolfe, J. Electron. Mater. 28(10), L24-L26 (1999).

${ }^{10}$ K. Cui, S. Fathololoumi, M. G. Kibria, G. A. Botton, and Z. Mi, Nanotechnology 23, 085205 (2012).

${ }^{11}$ Y.-L. Chang, F. Li, A. Fatehi, and Z. Mi, Nanotechnology 20, 345203 (2009).

${ }^{12}$ D. Wang, A. Pierre, M. G. Kibria, K. Cui, X. Han, K. H. Bevan, H. Guo, S. Paradis, A.-R. Hakima, and Z. Mi, Nano Lett. 11, 2353-2357 (2011).

${ }^{13}$ Y. Huang, X. Duan, Y. Cui, and C. M. Lieber, Nano Lett. 2, 101-104 (2002).

${ }^{14}$ O. V. Bilousov, J. J. Carvajal, D. Drouin, X. Mateos, F. Díaz, M. Aguiló, and C. O’Dwyer, ACS Appl. Mater. Interfaces 4(12), 6927-6934 (2012).

${ }^{15}$ J. J. Carvajal, O. Bilousov, D. Drouin, M. Aguiló, F. Díaz, and J. C. Rojo, Microsc. Microanal. 18, 905 (2012).

${ }^{16}$ J. J. Carvajal and J. C. Rojo, Cryst. Growth Des. 9, 320 (2009).

${ }^{17}$ D. K. Schroder, Semiconductor Material and Device Characterization (Wiley, New York, 1998).

${ }^{18}$ J. O. Song, J.-S. Ha, and T.-Y. Seong, IEEE Electron Device Lett. 57(1), 42 (2010).

${ }^{19}$ Y. Yang, Y. Ling, G. Wang, X. Lu, Y. Tong, and Y. Li, Nanoscale 5(5), 1820-1824 (2013). 
${ }^{20}$ B. Santic, Semicond. Sci. Technol. 18, 219 (2003).

${ }^{21}$ F. A. Ponce, D. P. Bour, W. Gatz, and P. J. Wright, Appl. Phys. Lett. 68, 57 (1996).

${ }^{22}$ B. Qu, Q. Zhu, X. Sun, S. Wan, Z. Wang, H. Nagai, Y. Kawaguchi, K. Hiramatsu, and N. Sawaki, J. Vac. Sci. Technol. A 21(4), 838-841 (2003).

${ }^{23}$ M. A. Reshchikov and H. Morkoç, J. Appl. Phys. 97(6), 061301061301-061395 (2005).

${ }^{24}$ U. Kaufmann, M. Kunzer, M. Maier, H. Obloh, A. Ramakrishnan, B. Santic, and P. Schlotter, Appl. Phys. Lett. 72(11), 1326-1328 (1998).
${ }^{25}$ L. Eckey, J. C. Holst, P. Maxim, R. Heitz, A. Hoffmann, I. Broser, B. Meyer, C. Wetzel, E. Mokhov, and P. Baranov, Appl. Phys. Lett. 68(3), 415-417 (1996).

${ }^{26}$ J. Neugebauer and C. G. Van de Walle, Appl. Phys. Lett. 69(4), 503-505 (1996).

${ }^{27}$ O. Gelhausen, H. Klein, M. Phillips, and E. Goldys, Appl. Phys. Lett. 83(16), 3293-3295 (2003).

${ }^{28}$ K. Saarinen, T. Laine, S. Kuisma, J. Nissilä, P. Hautojärvi, L. Dobrzynski, J. M. Baranowski, K. Pakula, R. Stepniewski, M. Wojdak, A. Wysmolek, T. Suski, M. Leszczynski, I. Grzegory, and S. Porowski, Phys. Rev. Lett. 79(16), 3030-3033 (1997). 